\title{
Elastoviscoplastic micromechanical modeling of the transient creep of ice
}

\author{
O. Castelnau, ${ }^{1,2}$ P. Duval, ${ }^{3}$ M. Montagnat, ${ }^{3}$ and R. Brenner ${ }^{1}$
}

Received 15 April 2008; revised 17 July 2008; accepted 28 July 2008; published 22 November 2008.

[1] A salient feature of the rheology of isotropic polycrystalline ices is the decrease of the strain rate by more than 2 orders of magnitude during transient creep tests to reach a secondary creep regime at a strain which is systematically of $\sim 1 \%$. We use a recent (socalled "affine") version of the self-consistent mean-field theory to model the elastoviscoplastic behavior of ice. The model aims at bridging scales between the rheology of single grain and the one of polycrystals by evaluating the intergranular interactions. It takes into account the long-term memory effects, which manifests itself by the fact that local stress and strain rate in grains depend on the whole mechanical history of the polycrystal. It is shown that the strong hardening amplitude during the transient creep is entirely explained by the stress redistribution within the specimen, from an almost uniform stress distribution upon instantaneous loading (purely elastic response) to strong interphase and intraphase heterogeneities in the stationary regime (purely viscoplastic response). The experimental hardening kinetic is much too slow to be explained by the same process; it is attributed to the hardening of hard glide slip systems (prismatic slip) in the transient regime. Moreover, the model very well reproduces the permanent creep rate of several highly anisotropic specimens of the Greenland Ice Core Project ice core (pronounced crystallographic textures), when accounting for a single-grain rheology that well matches the experimental one. Our results are consistent with recent findings concerning dislocation dynamics in ice.

Citation: Castelnau, O., P. Duval, M. Montagnat, and R. Brenner (2008), Elastoviscoplastic micromechanical modeling of the transient creep of ice, J. Geophys. Res., 113, B11203, doi:10.1029/2008JB005751.

\section{Introduction}

[2] There are significant issues in Geophysics related to the elastoviscoplastic behavior of polycrystalline materials such as ice and minerals. One can evoke for example the postseismic deformations in fault regions due to the viscoelastic behavior of the lower crust and/or the upper mantle [Kenner and Segall, 2000; Barbot et al., 2008], the uplift of continents associated to postglacial rebound [Yuen et al., 1986], the size-dependent relaxation of impact basin on the Moon and on Mars [Mohit, 2008], the attenuation of seismic waves associated to viscous dissipation along the travel path of the wave [Karato and Spetzler, 1990], and more generally all situations in which the mechanical response of the material involves both elasticity and viscoplasticity. For ice, transient effects associated to the elastoviscoplastic behavior should be encountered when ice flow changes direction rapidly, such as for glaciers flowing above irregular bed

\footnotetext{
${ }^{1}$ Laboratoire des Propriétés Mécaniques et Thermodynamiques des Matériaux, CNRS, Université Paris Nord, Villetaneuse, France.

${ }^{2}$ Institution of Geophysics and Planetary Physics, Scripps Institution of Oceanography, University of California, San Diego, La Jolla, California, USA.

${ }^{3}$ Laboratoire de Glaciologie et Géophysique de l'Environnement, CNRS, Université Joseph Fourier, Saint-Martin d'Hères, France.

Copyright 2008 by the American Geophysical Union. 0148-0227/08/2008JB005751\$09.00
}

rocks or for sea ice coming up against an offshore rig. Besides geophysical issues, the transient creep response of ice is highly valuable for the understanding of the elementary deformation mechanisms, such as glide and climb of dislocations, as it will be shown here.

[3] During the last two decades, significant efforts have been made for the development of physically based micromechanical models, aiming at estimating the overall behavior of polycrystalline materials from the knowledge of their microstructure (such as the lattice preferred orientation, LPO) and the elementary deformation mechanisms at the grain scale. However, these models have been applied in Geophysics to predict independently the overall purely elastic or the overall purely viscoplastic behaviors. In the purely elastic domain, applications include for example predictions of the seismic wave anisotropy as function of the strength of the LPO, with implications for the upper mantle structure [Mainprice, 1997; Blackman, 2007]. As for the purely viscoplastic regimes, applications include the predictions of LPO development and associated viscoplastic anisotropy in minerals [Dawson and Wenk, 2000] and ice [Castelnau et al., 1996] when the material is subjected to large plastic strain, with implications for the large-scale flow in the mantle or in ice sheets [Mangeney et al., 1996; Mangeney and Califano, 1998]. But, it is emphasized that the elastoviscoplastic case (i.e., when elastic and viscoplastic strain rates are present simultaneously in the mate- 
rial) has received much less attention owing to some specific difficulties, as we will detail here.

[4] Transient creep of polycrystalline ice has been the object of extensive experimental studies. For deformations at constant stress and temperature, it can be reasonably well described by the phenomenological relation [Andrade, 1910]

$$
\varepsilon=\varepsilon_{0}+\beta t^{1 / 3}+\kappa t
$$

where $\varepsilon_{0}$ is the instantaneous elastic strain, $\beta t^{1 / 3}$ gives the time development of the transient (also called "primary") creep regime, and $\kappa$ represents the steady state (viscoplastic) strain rate associated to the secondary (or "permanent", or "stationary") regime. This behavior has been observed in many other materials including noncrystalline ones [Cottrell, 1996; Dysthe et al., 2002]. Andrade creep has not been yet adequately explained, despite its general nature. It is associated with the collective dislocation motion in a long-range internal stress field and seems to be related to the existence of the critical point behavior [Cottrell, 1996, 1997]. In polycrystalline ice, strain rate decreases by more than 2 orders of magnitude during the transient regime [Jacka, 1984], and, at relatively low temperature, the secondary creep regime corresponds to a true steady state as soon as dynamic discontinuous (also called "migration") recrystallization is prevented [Jacka and Jun, 2000]. A striking feature of the deformation of ice is that a significant part of this transient creep is recoverable, i.e., an elongation of initially compressed specimens is observed when the macroscopic stress is suppressed (Figure 1). This reverse strain gives evidence for a kinematic hardening, directly caused by the development of long-range internal stress field in the specimen [Ashby and Duval, 1985].

[5] In ice as in most polycrystalline materials, individual grains exhibit anisotropic mechanical behaviors. Each grain thus reacts differently to the imposed stress, depending on its lattice orientation, but since the cohesion of the polycrystalline material has to be maintained, strong mechanical interactions between grains must build up, leading to highly heterogeneous stress and strain rate distributions inside grains and between different grains. This heterogeneity has a strong influence on the overall behavior of the material [Castelnau et al., 2006], since elementary deformation mechanisms such as dislocation glide are activated with respect to the local stress level. In ice, individual crystals exhibit a relatively low elastic anisotropy, but a very large viscoplastic anisotropy owing to the presence of only two independent easy slip systems (basal plane) for the dislocations. Therefore, the stress distribution is expected to be significantly different for purely elastic and purely viscoplastic regimes, with a much higher stress heterogeneity for the latter. During transient creep experiments, a strong evolution of the stress field must thus occur since the material evolves from a purely elastic response at $t=0$ to a purely viscoplastic one as $t \rightarrow \infty$, as in equation (1). This redistribution of stress may be at the origin of the very large decrease of strain rate during the transient creep [Duval et al., 1983; Ashby and Duval, 1985].

[6] The objective of this work is to obtain an accurate and physically based description of the creep behavior of polycrystalline ice. Emphasis is placed on the evolution of the intergranular interactions in the transient regime, and subsequent stress and strain rate heterogeneities inside and between grains. For doing this, we apply a micromechanical model based on a mean-field homogenization approach, that accounts for the elastoviscoplastic behavior of individual grains (including the observed slip systems for the dislocations) and for the coupling between elasticity and viscoplasticity that gives rises to the so-called "long-term memory effect" as explained below. Therefore, after a description of the elastic and viscoplastic behavior of ice single crystals and polycrystals (section 2 ), the constitutive relation for single crystals is specified (section 3 ). The very general features of the micromechanical model are introduced in section 4, with some more details given in the appendix. Results concerning the effective behavior during transient creep are given in section 5, and later discussed with emphasis on field statistics at the level of grains. Prediction for overall anisotropy in the permanent creep regime will also be discussed.

\section{Experimental Deformation Behavior of Ice}

\subsection{Elastic Behavior of Ice Crystals}

[7] Ice $1 \mathrm{~h}$ belongs to the space group P63/mmc. Owing to its transverse isotropy, the elastic behavior of ice single crystals is described by a set of five elastic moduli. Values of these moduli have been determined on several artificial and natural single crystals by Gammon et al. [1983]. The elastic anisotropy of single crystals is small, the Young's modulus $E$ varying by about $30 \%$ depending on the direction of loading with respect to the $c$ axis. The highest value is along the $c$ axis with $E=11.8 \mathrm{GPa}$ at $-16^{\circ} \mathrm{C}$. Variation with temperature is unimportant for conditions prevailing in ice sheets and glaciers, with elastic constants varying typically by about $1.5 \%$ for a temperature variation of $5^{\circ} \mathrm{C}$.

\subsection{Viscoplastic Behavior of Ice Crystals}

[8] The main feature of the plasticity of the ice crystal is its anisotropy. For shear stresses of the order of $0.1-1 \mathrm{MPa}$, ice deforms easily by shear on the basal plane, on the systems $\{0001\}\langle 11 \overline{2} 0\rangle$, which provide two independent deformation systems. The creep curves of single crystals oriented for basal slip show an accelerating creep up to a strain higher than 10\% [Griggs and Coles, 1954], which is explained by the multiplication of dislocations during transient creep [Higashi et al., 1964]. Although there is no evidence that a real steady state was reached in laboratory creep tests, a stress sensitivity (or stress exponent) $n \approx 2$ is generally adopted, with an activation energy close to $60 \mathrm{~kJ} \mathrm{~mol}^{-1}$ [Duval et al., 1983].

[9] When ice crystals are loaded such that there is no resolved shear stress on the basal plane, creep rate are so small that it is doubtful that they have ever been measured. Any slight misorientation of the $c$ axis with respect to the direction of the applied stress results in the activation of basal slip. The resistance to shear on nonbasal planes can be 60 times higher than resistance on the basal plane [Duval et al., 1983].

[10] Multiplication of dislocations during transient creep was explained by the climb of $60^{\circ}$ basal dislocations on the $\{1 \overline{1} 00\}$ prismatic planes [Ahmad and Whitworth, 1988]. 


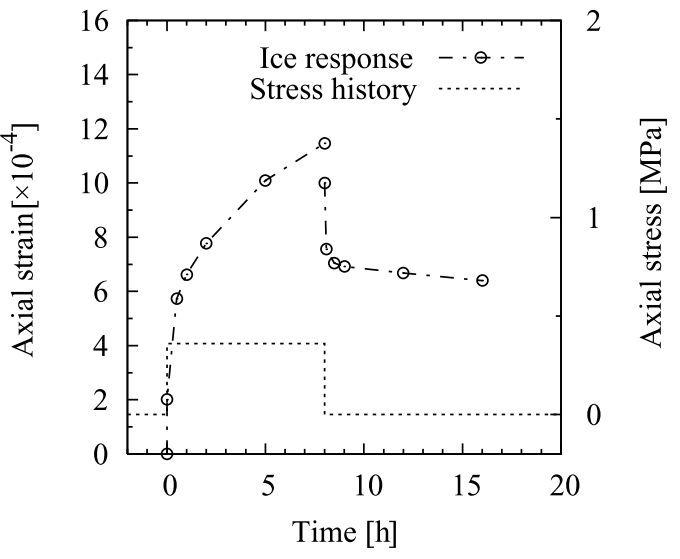

Figure 1. Elastoviscoplastic response of an isotropic specimen of ice (grain size $5 \mathrm{~mm}$ ) deformed at $-1.5^{\circ} \mathrm{C}$. A uniaxial constant stress $(0.36 \mathrm{MPa})$ is applied for $0 \leq t<8 \mathrm{~h}$. When suppressing the load at $t=8 \mathrm{~h}$, a significant recovery creep is observed. For comparison, the purely elastic deformation for this stress level is $\sim 3.8 \times 10^{-5}$. Data are from Duval [1976].

The cross slip of basal screw dislocations was recently invoked to explain dislocation multiplication and the scaleinvariant character of the dislocation arrangement observed in single crystals deformed under torsion [Montagnat et al., 2006]. The dissociation of screw dislocations on the basal plane [Hondoh et al., 1990] would not prevent this cross slip since the constriction of partial dislocations close to jogs should preexist [Friedel, 1964].

[11] Then, the glide of nonbasal edge dislocations on prismatic planes, which would be produced through the climb of basal dislocations as well as the cross slip of basal dislocations on prismatic and pyramidal planes, give some consistency to the introduction of these deformation slip systems in the constitutive relation that will be used to simulate the behavior of the polycrystal (section 3). Obviously, considering the large viscoplastic anisotropy of the ice crystal, the contribution of the nonbasal slip systems to the deformation of the ice crystal must be very low.

\subsection{Viscoplastic Behavior of Polycrystals}

[12] The creep behavior of isotropic (i.e., with random LPO) polycrystalline ice is well documented [e.g., see Jacka, 1984; Alley, 1992]. During the primary or transient creep, strain rate decreases with time by a factor that can be higher than 100 to reach a minimum value corresponding to the secondary creep for a strain of about 1\% [Jacka, 1984]. According to Ashby and Duval [1985], the stress state within the polycrystal may be close to uniform on first loading. But, as grain boundaries act as obstacles to basal slip, creep relaxes the resolved stress on the basal plane on each grain and the load is transferred to the harder nonbasal slip systems. Transient creep is therefore associated with the development of a long-range internal stress field, which is at the origin of a significant time-dependent recoverable strain when the load is removed [Duval, 1978].

[13] For deviatoric stresses higher than $0.2 \mathrm{MPa}$, the stress exponent of the secondary creep, corresponding to the minimum creep rate, is close to 3 [Duval et al., 1983]. Deformation is essentially produced by basal slip, which contributes to the total deformation by more than $80 \%$ [Castelnau et al., 1997; Lebensohn et al., 2007]. It is worth noting that polycrystalline ice cannot be deformed to large strains by basal slip only. Although five independent slip systems may be required to accommodate any arbitrary viscoplastic deformation, it has been shown that four independent systems are in fact sufficient for polycrystalline ice [Hutchinson, 1977; Nebozhyn et al., 2000; Lebensohn et al., 2007]. Basal slip could be accommodated by prismatic slip, the climb of edge segments of basal dislocations, and/ or pyramidal slip, as evoked in section 2.2. It is worth noting that intracrystalline strain heterogeneities, associated with the long-range internal stress field, should contribute to reduce the activity of nonbasal slip systems.

[14] The increase of the strain rate in the tertiary creep regime initiated for a strain higher than $1 \%$ is explained by the occurrence of dynamic migration recrystallization [Steinemann, 1958]. This recrystallization regime is generally observed in glaciers at high temperature as well as in most laboratory experiments [Gow and Williamson, 1976; Duval and Castelnau, 1995; Jacka and Jun, 2000] and is associated with the development of recrystallization LPOs. The stress exponent for the tertiary creep regime is higher than 3 [Steinemann, 1958]. A value of $\sim 4$ was obtained by Kirby et al. [1987].

[15] For conditions prevailing in polar ice sheets with deviatoric stresses lower than $0.1 \mathrm{MPa}$, laboratory and field observations support a stress sensitivity $n$ lower than 2 [Mellor and Testa, 1969; Dahl-Jensen and Gundestrup, 1987; Duval and Castelnau, 1995]. Furthermore, a newtonian viscosity was also deduced from densification data on glacier bubbly ice [Lipenkov et al., 1997]. According to Goldsby and Kohlstedt [2001], grain boundary sliding would be the dominant deformation mode in this stress regime and could accommodate basal slip. However, from observations of the evolution of the grain microstructure and LPOs along deep ice cores, the relatively low value of $n$ at low deviatoric stresses would be rather the result of the occurrence of dynamic rotation recrystallization as an accommodation process of basal slip [Montagnat and Duval, 2000]. With this recrystallization mechanism, grain boundaries migrate in the same low-velocity regime as the one associated with normal grain growth [Alley, 1992] and LPOs develop by the lattice rotation due to intracrystalline slip [Castelnau et al., 1996].

\section{Constitutive Relation for Ice Crystals}

[16] Our aim is to introduce the above mentioned elementary deformation mechanisms in the local constitutive relation of the model, in view of reaching a physically based model for the polycrystal behavior. Thus, to describe the transient effects in polycrystals, both the elastic and viscoplastic responses of ice crystals need to be accounted for. The total strain rate tensor $\dot{\varepsilon}(\mathbf{x}, t)$ at the spatial position $\mathbf{x}$ (i.e., inside a grain) and time $t$ expresses

$$
\dot{\varepsilon}(\mathbf{x}, t)=\dot{\varepsilon}^{\mathrm{el}}(\mathbf{x}, t)+\dot{\boldsymbol{\varepsilon}}^{\mathrm{vp}}(\mathbf{x}, t) .
$$


Table 1. Single Crystal Rheology Considered in the Model ${ }^{\mathrm{a}}$

\begin{tabular}{lccccc}
\hline & Slip System & $n$ & $\tau^{\text {init }}$ & $\tau^{\text {sta }}$ & $H$ \\
\hline $\mathrm{Ba}$ & $\{0001\}\langle 11 \overline{2} 0\rangle$ & 2.00 & 0.125 & 0.035 & -25 \\
$\mathrm{Pr}$ & $\{1 \overline{1} 00\}\langle 11 \overline{2} 0\rangle$ & 2.85 & 0.21 & 1.30 & 120 \\
$\mathrm{Pyr}$ & $\{11 \overline{2} 2\}\langle 11 \overline{2} \overline{3}\rangle$ & 4.00 & 3.0 & 3.10 & 120 \\
\hline
\end{tabular}

${ }^{a}$ Slip plane $\{h k i l\}$ and orientation of the Burgers vector $\langle u v t w\rangle$ of the basal $(\mathrm{Ba})$, prismatic $(\mathrm{Pr})$, and pyramidal (Pyr) slip systems. The stress sensitivity $n$, the initial (init) and stationary (sta) values of the reference shear stresses $\tau^{(0)}$, and the hardening coefficient $H$ have been obtained by identification on experimental data; see text for details. Values of $\tau$ and $H$ are given in $\mathrm{MPa}$, for a temperature of $-10^{\circ} \mathrm{C}$.

The elastic part is related to the stress rate

$$
\dot{\varepsilon}^{\mathrm{el}}(\mathbf{x}, t)=\mathbf{S}^{(r)}: \dot{\boldsymbol{\sigma}}(\mathbf{x}, t)
$$

with $\mathbf{S}^{(r)}$ the elastic compliance tensor, and the colon denoting the double contracted product (for example, the tensor $\dot{\varepsilon}=\mathbf{S}$ : $\dot{\boldsymbol{\sigma}}$ has components $\left.\dot{\varepsilon}_{i j}=\sum_{k l} S_{i j k l} \dot{\sigma}_{k l}\right)$. The subscript $(r)$ refers to the lattice orientation of the grain present at the spatial position $\mathbf{x}$. Concerning the viscoplastic response, we consider that deformation occurs only by dislocation glide on a given number of slip systems. Note that climb and cross slip of basal dislocations are not introduced here, since this would require theoretical and numerical developments far beyond the scope of this paper. The resolved shear stress $\tau_{(k)}$ acting on a slip system $(k)$ is given by a projection of the local deviatoric stress tensor $\boldsymbol{\sigma}^{\prime}$

$$
\tau_{(k)}(\mathbf{x}, t)=\boldsymbol{\mu}_{(k)}^{(r)}: \boldsymbol{\sigma}^{\prime}(\mathbf{x}, t)
$$

with $\boldsymbol{\mu}_{(k)}^{(r)}$ the Schmid tensor expressing the orientation of the slip system with respect to a laboratory reference frame. The slip rate $\dot{\gamma}$ on system $(k)$ is further expressed by a standard power law

$$
\dot{\gamma}_{(k)}(\mathbf{x}, t)=\dot{\gamma}_{0}\left|\frac{\tau_{(k)}(\mathbf{x}, t)}{\tau_{(k)}^{0(r)}(t)}\right|^{n_{(k)}-1} \frac{\tau_{(k)}(\mathbf{x}, t)}{\tau_{(k)}^{0(r)}(t)}
$$

with $\tau_{(k)}^{0(r)}$ a reference shear stress that expresses the resistance of system $(k)$ in the lattice orientation $(r)$ at time $t, n_{(k)}$ the stress sensitivity, and $\dot{\gamma}_{0}$ a reference slip rate taken here equal to $10^{-6} \mathrm{~s}^{-1}$. Combining all available slip systems, the viscoplastic strain rate thus reads

$$
\dot{\boldsymbol{\varepsilon}}^{\mathrm{vp}}(\mathbf{x}, t)=\sum_{k=1}^{K} \boldsymbol{\mu}_{(k)}^{(r)} \dot{\gamma}_{(k)}(\mathbf{x}, t)
$$

with $K$ the total number of slip systems. As deformation proceeds, the reference shear stresses evolve owing to the evolution of the dislocation density (typically, $\tau_{(k)}^{0(r)}$ is considered to be proportional to the square root of the dislocation density [e.g., see Kocks, 1976], although we will not go that deep in the physical interpretation here). We consider a simple and standard form for the hardening/ softening law [Bronkhorst et al., 1992; Castelnau et al., 2001]

$$
\dot{\tau}_{(k)}^{0(r)}=\sum_{l} H_{(k)} \frac{\tau_{(l)}^{\text {sta }}-\tau_{(l)}^{0(r)}}{\tau_{(l)}^{\text {sta }}-\tau_{(l)}^{\text {init }}}\left|\left\langle\dot{\gamma}_{(l)}\right\rangle^{(r)}\right|
$$

with $\langle.\rangle^{(r)}$ denoting the average over the volume of grains with lattice orientation $(r)$. The coefficient $H_{(k)}$ expresses the bulk hardening rate. Starting with an initial value $\tau_{(k)}^{\text {init }}$ before loading (i.e., $t=0$ ), the reference shear stress of a system $(k)$ evolves with strain to reach the final value $\tau_{(k)}^{\text {sta }}$ in the stationary regime (permanent creep, $t \rightarrow \infty$ ). Equation (7) allows the reference stress of each slip system and each grain orientation to evolve independently, depending on the local pattern of plastic deformation.

[17] The slip systems considered here are indicated in Table 1. Besides the easy basal glide, prismatic slip is considered according to experimental observations of dislocation structures. Although these four independent slip systems are theoretically sufficient to allow general deformation, we introduce a third slip mode (pyramidal slip) for the sake of allowing axial deformation along the $c$ axes. The relevance of this last system will be discussed later in this paper.

[18] Part of the local constitutive relation can be identified by considering experimental data on single crystals. The elastic constants used in this work are those given by Gammon et al. [1983]. Concerning the viscoplastic regime, only experimental data promoting the activation of basal slip are really reliable. The stress sensitivity $n_{(\mathrm{Ba})}$ and the stationary reference stress $\tau_{(\mathrm{Ba})}^{\mathrm{sta}}$ for basal slip have been determined at $-10^{\circ} \mathrm{C}$ based on the experimental data in permanent creep regime compiled by Duval et al. [1983] and reproduced in Figure 10. The two remaining coefficients, $\tau_{(\mathrm{Ba})}^{\text {init }}$ and $H$, have been identified on the transient creep response of ice single crystal given by Weertman [1973]. The obtained values are reported in Table 1. We obtained a negative value for $H_{(\mathrm{Ba})}(-25 \mathrm{MPa})$ owing to the softening of basal creep during the transient regime. The obtained creep behavior is compared to experimental data in Figure 2, for a wide range of applied strain rates (3 orders of magnitude). It is observed that in spite of its simple form, the evolution law (7) matches the data reasonably well, with the stress peak characteristic of the softening of basal slip.

\section{Micromechanical Modeling}

[19] To estimate the intergranular interactions during transient creep, and their impact on the effective behavior of polycrystalline ice, a micromechanical model is required. The mechanical problem to be solved is that of a continuous material (polycrystalline ice), with different elastoviscoplastic behavior in the different grains (since they exhibit different lattice orientation), and submitted to a constant and uniform macroscopic stress. Furthermore, the physical deformation mechanisms at the grains scale (dislocation glide), introduced above, and the arrangement of grains in the specimen (in particular, the LPO), have to be taken into account. A convenient way to tackle the problem is to make use of mean-field homogenization methods, which provide a solution of the problem based on a statistical description 


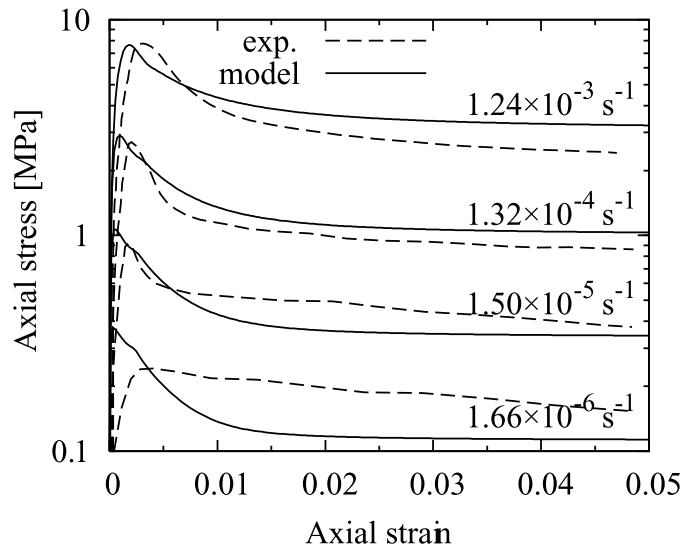

Figure 2. Behavior of ice single crystal given by equations (2)-(7) for a deformation by basal glide and compared to the experimental data given by Weertman [1973]. The $c$ axis is oriented at $45^{\circ}$ from the uniaxial compression direction. Applied axial strain rates are indicated. The temperature is $-10^{\circ} \mathrm{C}$.

of the material microstructure. In this context, the selfconsistent (SC) scheme, initially proposed to deal with the elasticity of polycrystals [Hershey, 1954; Kröner, 1958, 1978; Willis, 1981], has been further extended (in different ways) to deal with purely viscoplastic responses. For example, the "tangent" extension of the SC scheme [Molinari et al., 1987; Lebensohn and Tomé, 1993] has been applied to describe the viscoplastic anisotropy of polar ices with pronounced LPO by Castelnau et al. [1996, 1997, 1998].

[20] The treatment with the same method of the elastoviscoplasticity of polycrystals is somehow more complicated, see for instance [Laws and McLaughlin, 1978]. In short, it can be shown that even in the simple case of a polycrystal comprising grains whose behavior exhibits a single relaxation time (so-called "short term memory"), the effective behavior exhibits a continuous spectrum of relaxation time ("long memory effect") [Sanchez-Hubert and SanchezPalencia, 1978; Suquet, 1987]. A consequence of this feature is that the overall strain rate of the polycrystal (and that of the grains inside) at a given time $t$ depends on the whole strain history of the specimen between times 0 and $t$. To obtain the exact effective mechanical response at time $t$, it is thus required to keep track of an infinite number of information (or internal variables) corresponding to the overall strains at all previous times, and therefore the problem is not simple. Within mean-field approaches, some approximations (with hopefully limited effects on the accuracy of the solution) are thus necessary. Basically, two approaches have been proposed to deal with this problem. The recent method proposed by Lahellec and Suquet [2006, $2007 \mathrm{a}$, based on an incremental variational procedure, is worth mentioning, but it has been applied up to now only to simple two-phases composite materials. The approach used in the sequel, which provides a good compromise between accuracy of the solution and simplicity of the formalism, is the so-called "affine" Self-consistent method due to Masson and Zaoui [1999]. It is based on the correspondence principle [Mandel, 1966] which indicates that the elastoviscoplastic problem can be reduced to a simpler homogenization problem if solved in the Laplace space. This approach has already given promising results as for the creep behavior of zirconium alloys [Letouzé et al., 2002; Brenner et al., 2002a], since it retains the long-term memory effect associated with the elastoviscoplastic coupling. We recall in the Appendix, for completeness of this paper, the main ingredients of this model.

\section{Results and Discussion}

[21] The affine SC micromechanical model is now applied to ice polycrystals. Results presented below are obtained for a temperature of $-10^{\circ} \mathrm{C}$. There are few parameters $\left(\tau_{(.)}^{\text {init }}, \tau_{(.)}^{\text {sta }}, n_{(.)}, H_{(.)}\right)$in the model that needs to be determined. All of these are associated with the elementary deformation mechanisms at the local (grain) scale. Since we expect the model to provide a physically based mechanical description of polycrystalline ice, i.e., a correct bridging between the effective behavior of the polycrystal and the deformation mechanisms at the grain scale, the model should be able not only to reproduce transient regimes, but also to provide accurate results for permanent creep rates. In particular, it should estimate correctly the anisotropy of polar ices with pronounced LPO.

[22] For this first application, we choose to proceed in two steps. First, we identify the four parameters involved in the secondary creep (purely viscoplastic) regime. To obtain reliable values, we need to use data for specimens exhibiting different microstructures and deformed under different conditions, such as those available for polar ices with various LPO. Then, transient regimes of isotropic specimens will be investigated, and results will be discussed in terms of active deformation mechanisms and stress/strain heterogeneities inside and between grain orientations.

\subsection{Rheology and Anisotropy for Secondary Creep}

[23] The stationary reference shear stresses $\tau_{(\operatorname{Pr})}^{\mathrm{sta}}$ and $\tau_{(\mathrm{Pyr})}^{\mathrm{sta}}$ and stress sensitivities $n_{(\mathrm{Pr})}$ and $n_{(\mathrm{Pyr})}$ of the prismatic and pyramidal systems have been identified to reproduce the purely viscoplastic response of various isotropic and anisotropic specimens. For isotropic ice, we considered the data compiled by Duval et al. [1983] which lead, for stresses between 0.2 and $2 \mathrm{MPa}$, to the generally adopted value $n=3$ for the macroscopic stress sensitivity (Figure 3 ). As for the anisotropic specimens, we used the data of Castelnau et al. [1998] obtained on specimens from the Greenland Ice Core Project (GRIP) ice core (central Greenland). With increasing depth, randomly oriented $c$ axis at the surface of the ice sheet tend to concentrate toward the in situ vertical direction up to a depth of $\sim 2600 \mathrm{~m}$. Beneath this depth, less pronounced LPOs are observed because of the initiation of migration recrystallization [Thorsteinsson et al., 1997]. Castelnau et al. [1998] obtained the experimental stationary creep behavior of GRIP ices for two loading conditions, reported in Figure 3. The first one corresponds to an in situ vertical compression, showing an increasing flow stress (decreasing strain rate) with increasing depth, since the activation of secondary slip systems is favored for pronounced LPO. The second loading condition corresponds to in situ horizontal shear, promoting basal slip 

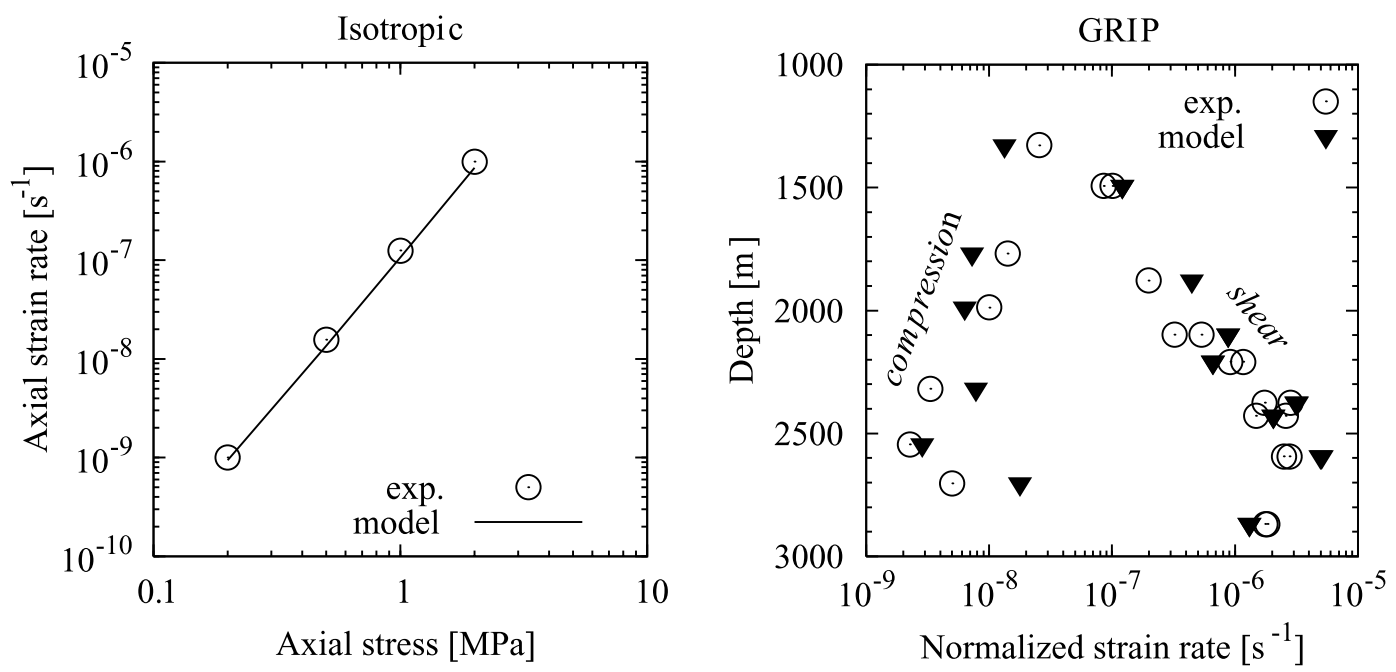

Figure 3. Stationary creep behavior at $-10^{\circ} \mathrm{C}$ calculated by the affine $\mathrm{SC}$ model, and compared to experimental data. (left) Isotropic specimens. Experimental data correspond to the mean behavior indicated by Duval et al. [1983]. (right) Anisotropic specimens from the GRIP ice core, exhibiting a LPO with an increasing concentration of $c$ axes toward the vertical direction from the surface of the ice sheet down to $\sim 2600 \mathrm{~m}$ depth, and a decrease below due to dynamic recrystallization. Experimental data from Castelnau et al. [1998] are expressed for a stress of $1 \mathrm{MPa}$ using a stress sensitivity $n=3$. Points on the left-hand side reflect the (hard) behavior under vertical compression, whereas data on the right correspond to (soft) horizontal shear.

and resulting in a softening of the ice with increasing LPO strength.

[24] Values for the reference shear stresses and stress sensitivities for the secondary slip systems, identified using this data set, are given in Table 1. Pyramidal slip is found to be much more difficult than prismatic slip, a result which seems in qualitative agreement with the known dislocation structure in ice. In Figure 3, the effective behavior deduced by the model is compared to the experimental data. For isotropic specimens, the overall flow stress is perfectly reproduced. It is also remarkable that a constant macroscopic stress sensitivity $n=3$ is obtained for a relatively wide range of stress, even with significantly different values at the slip system level (Table 1). The agreement is also excellent with the anisotropic specimens of the GRIP ice core, meaning that the relation between LPO and effective rheology is very well captured. The model nicely reproduces the increasing anisotropy from the surface down to $2600 \mathrm{~m}$ depth, and the decreasing one below. The difference by more than 2 orders of magnitude between the vertical and shear strain rates at $\sim 2600 \mathrm{~m}$ is also perfectly reproduced, although this was a challenging feature for the model. On the basis of the large variety of LPO and loading conditions used for this identification, it is expected that the obtained parameters are physically relevant for the viscoplastic response at the scale of grains.

\subsection{Transient Creep Behavior of Isotropic Ice}

[25] The affine SC model is now applied to the transient creep behavior of isotropic specimens, involving thus both elasticity and viscoplasticity at the grain scale. We use for this the data set compiled by Ashby and Duval [1985]. The original data are expressed in adimensional axes, the axial strain rate being normalized by the minimum strain rate obtained for the stationary creep regime, and the axial strain being normalized by the applied stress divided by the Young's modulus $E$ ( $E \simeq 9330 \mathrm{MPa}$ for polycrystalline ice). For the sake of clarity, the same data are converted at a temperature of $-10^{\circ} \mathrm{C}$ and an applied stress of $1 \mathrm{MPa}$ (see Figure 4). Note in passing that the scaling proposed by Ashby and Duval [1985] may not be completely universal since it is not strictly followed by the SC scheme.

[26] First of all, transient creep behavior has been calculated with the affine SC model assuming that there is no

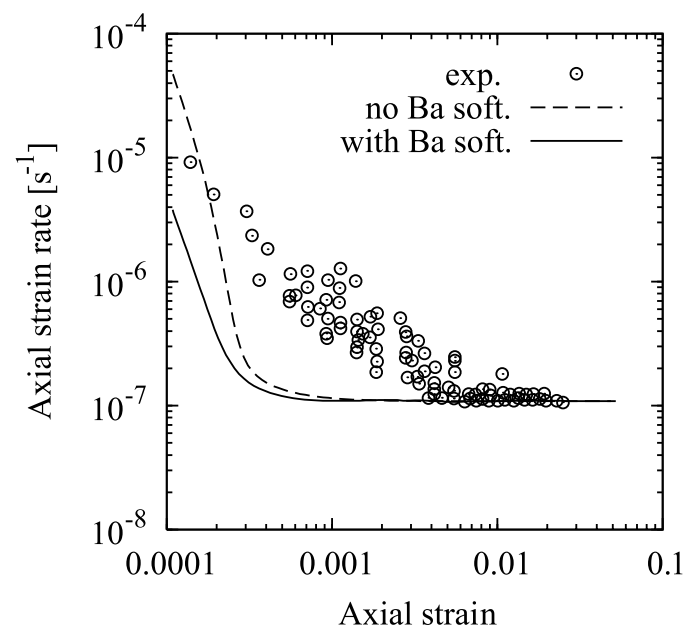

Figure 4. Transient creep response of isotropic ice under a uniaxial compressive stress of $1 \mathrm{MPa}$. Model results are shown with and without taking into account the softening of basal slip during deformation. Results are compared to the data of Ashby and Duval [1985], expressed for the same loading conditions. 

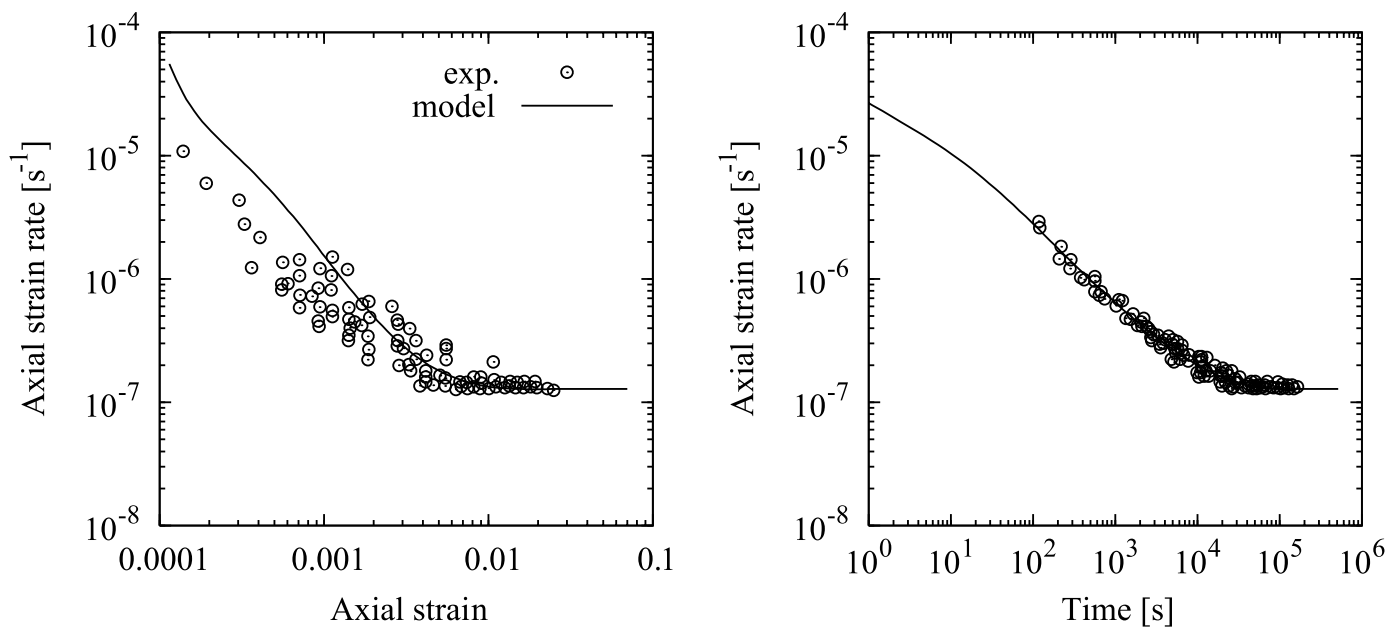

Figure 5. Transient creep response of isotropic ice under a uniaxial compressive stress of 1 MPa. Model results are compared to the data of Ashby and Duval [1985], expressed for the same loading conditions. Strain hardening of prismatic and pyramidal slip systems is taken into account.

evolution of the reference shear stresses during the deformation, for all systems. That is, only the stationary values identified above have been considered. Results are shown in Figure 4. It can be observed that the model nicely reproduces the very strong overall hardening of the specimen, with a strain rate decreasing by more than two orders of magnitude during the transient regime. However, stationary creep is obtained for a strain as small as $\sim 10^{-3}$, instead of $10^{-2}$ for the experimental data. Next, a second trial has been performed, with still no evolution for the reference shear stresses of the secondary systems, but accounting for the softening of basal slip in the transient regime, as described in section 3. Slight differences are obtained compared to the previous case, but the predicted overall hardening is even more rapid, resulting in a permanent creep regime appearing much too early.

[27] An evolution of the reference shear stresses for prismatic and pyramidal slip has thus to be accounted for to reproduce the experiments. Thus, $\tau_{(\mathrm{Pr})}^{\text {init }}, \tau_{(\mathrm{Pyr})}^{\text {init }}$, and $H$ have been identified on the same data set, keeping $\tau_{(\mathrm{Ba})}$ evolving as above. The corresponding values are reported in Table 1, and we obtained this time positive values for $H_{(\mathrm{Pr})}$ and $H_{(\mathrm{Pyr})}$ $(+120 \mathrm{MPa})$ indicating a hardening process. As shown in Figure 5 , the predicted behavior now perfectly matches with the data. The strain rate decreases by more than 2 orders of magnitude during transient creep, and the stationary regime is obtained at the correct strain of $\sim 1 \%$. Even better is the agreement when experimental data are plotted as strain rate versus time. Obviously, an increasing resistance to shear of the secondary systems is thus necessary to reproduce correctly the hardening kinetic at the polycrystal level. However, according to Table 1, this applies essentially to prismatic slip which reference stress varies by a factor $\sim 6$ during transient creep. As for the pyramidal systems, the evolution of the reference stress is probably insignificant $(\sim 3 \%)$. The same model results are shown in Figure 6 as total strain versus time. Interestingly, it can be observed that when plotted in logarithmic scale, an almost linear curve is obtained in the transient regime. The slope of this section is $\sim 0.38$, i.e., very close to that of Andrade's law (1) in which an exponent $1 / 3$ is generally considered.

\subsection{Field Statistics}

[28] Concerning transient creep, it has been shown in section 5.2 that the correct hardening amplitude is easily reproduced by the model as far as realistic elastic and viscoplastic rheologies are considered at the grain level. However, the hardening kinetic can only be explained if the strength of the secondary slip systems evolves with strain. The investigation of the distribution of stress and strain rate in the different grain orientations is of interest for a deeper understanding of this hardening process. Recall that the SC approach does not allow to consider stress and strain differences between individual grains; since all grains exhibiting the same lattice orientation are regrouped within

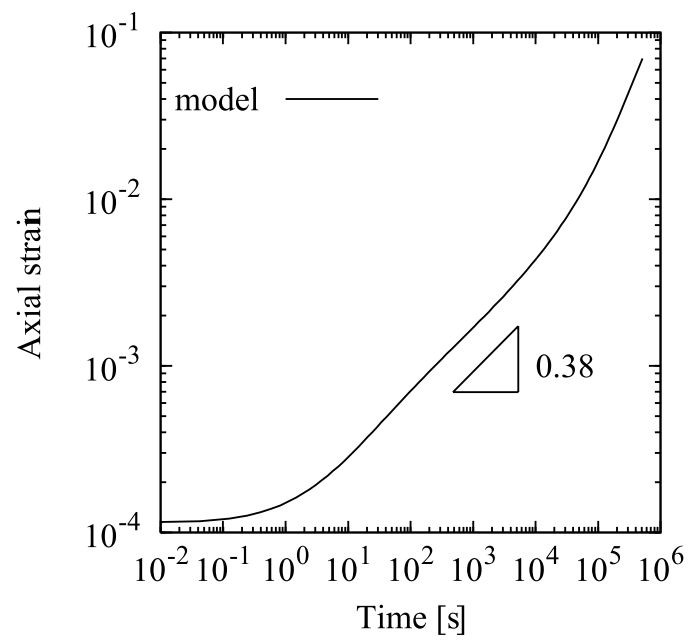

Figure 6. Same as Figure 5 but plotted in logarithmic scale as strain versus time. In the transient regime, the behavior is almost linear with a slope close to the one predicted by Andrade's law. 

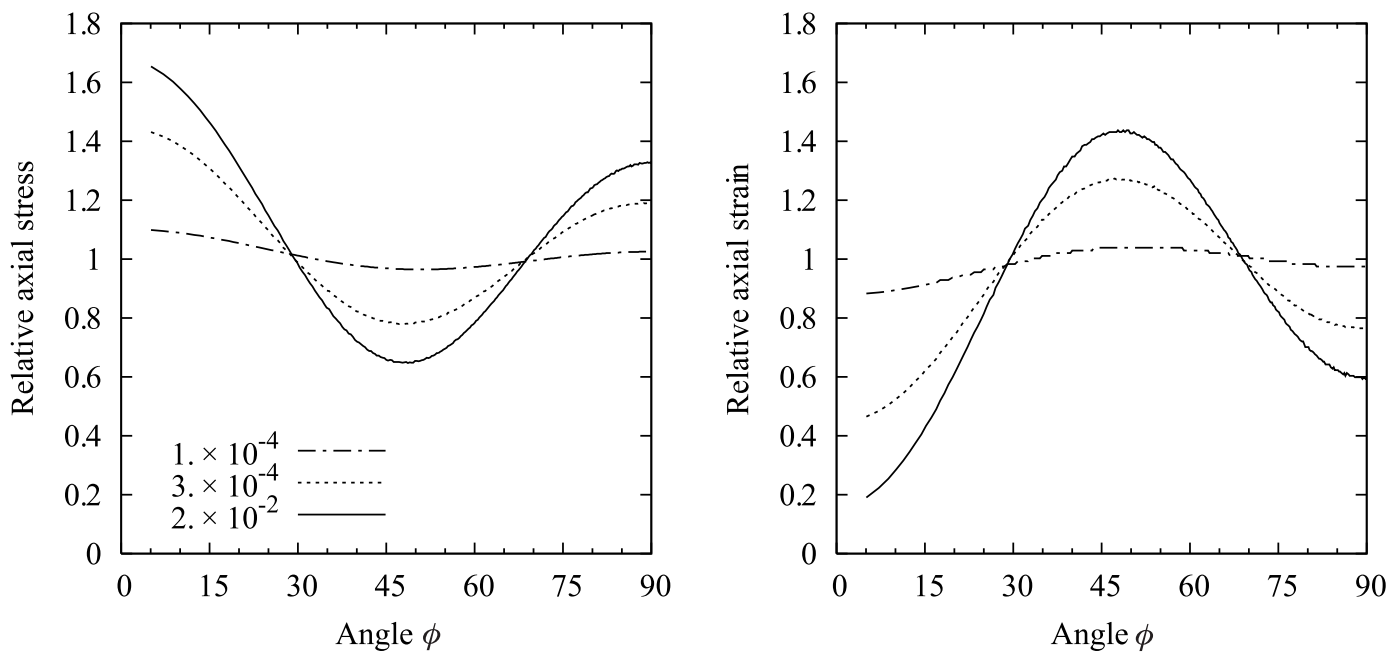

Figure 7. Distribution of the (normalized) phase average axial stress $\bar{\sigma}_{33}{ }^{(r)} / \bar{\sigma}_{33}$ and strain $\bar{\varepsilon}_{33}{ }^{(r)} / \bar{\varepsilon}_{33}$ with respect to the angle $\phi$ between the macroscopic compression direction and the $c$ axis, for times $0 \mathrm{~s}, 15 \mathrm{~s}$, and $1.5 \times 10^{5} \mathrm{~s}$, corresponding to macroscopic strains of $1.1 \times 10^{-4}$ (elastic response), $3 \times 10^{-4}$, and 2. $\times 10^{-2}$ (stationary creep) respectively.

a single mechanical phase, only the differences between those different phases (i.e., different orientations) can be investigated.

[29] In Figure 7, the distribution of the normalized average stress $\bar{\sigma}_{33}^{(r)} / \bar{\sigma}_{33}$ and strain $\bar{\varepsilon}_{33}^{(r)} / \bar{\varepsilon}_{33}$ are plotted with respect to the angle $\phi$ between the $c$ axis of grains and the direction of macroscopic compression $\mathbf{e}_{3}$. Here, $\bar{\sigma}_{33}^{(r)}$ denotes the average axial stress in grains with orientation $(r)$, and $\bar{\sigma}_{33}$ the average stress for the whole polycrystal (with a similar notation for the strain). We emphasize that $\bar{\sigma}_{33}^{(r)}$ and $\bar{\varepsilon}_{33}^{(r)}$ are phase average values, i.e., they can be thought as an integration over an infinite number of grains with identical lattice orientation $(r)$ but with different shape and surrounding. Results are shown at three stages: the instantaneous response upon loading, an intermediate stage in the transient regime, and the permanent creep regime. At $t=0 \mathrm{~s}$, only elastic deformation has occurred in grains, and the overall axial deformation $\left(1.1 \times 10^{-4}\right)$ only depends on the Young's modulus. It can be observed that both $\bar{\sigma}_{33}^{(r)} / \bar{\sigma}_{33}$ and $\bar{\varepsilon}_{33}^{(r)} / \bar{\varepsilon}_{33}$ weakly fluctuate with angle $\phi$, by not more than $\sim 15 \%$, indicating almost homogeneous stress and strain fields in the polycrystal. This result is attributed to the low elastic anisotropy of ice grains. On the other hand, results given for the stationary regime $\left(\bar{\varepsilon}_{33}=2 . \times 10^{-2}\right)$ indicate much larger fluctuations of stress and strain. Grains with $c$ axis at $\sim 45^{\circ}$ from the macroscopic compression axis are highly deformed but support a low stress level, whereas grains at $0^{\circ}$ or $90^{\circ}$ deform much less and at a high stress level. Note also that for the permanent creep, the strain rate distribution is (almost) identical to the strain distribution. Here, owing to the large viscoplastic anisotropy of grains, strong intergranular interactions build up during permanent creep. The deformation capability of grains is essentially driven by the possibility of soft slip systems to be activated. Grains at $\phi \simeq 0^{\circ}$ are found to be particularly hard to deform, with strain (rate) of about 1 order of magnitude smaller than the macroscopic counterpart. In those lattice orientations, one may expect pyramidal slip to be significantly activated, but, as shown by Castelnau et al. [2006, 2008b], local stress concentrations in strongly anisotropic polycrystals make the deformation pattern extremely dependent on the local grain configuration; soft slip systems (here basal slip) can be locally significantly activated even in grains exhibiting a vanishing Schmid factor (at $\phi=0^{\circ}$ ). This tendency is also confirmed by full-field computations [Lebensohn et al., 2007, 2008]. Grains at $\phi \simeq 90^{\circ}$ are in average slightly softer than those at $\phi \simeq 0^{\circ}$ due to smaller resistance prismatic slip as compared to pyramidal slip. Finally, results are further shown at $t=15 \mathrm{~s}$, i.e., for $\bar{\varepsilon}_{33}=3 . \times 10^{-4}$ which is about three times the purely elastic strain. Now, although the deformation is much smaller than that for stationary creep, the overall stress and strain distributions in the polycrystal obey very similar features as in the purely viscoplastic regime, i.e., a very large heterogeneity throughout the material with soft orientations at $\sim 45^{\circ}$ and hard ones at $0^{\circ}$ and $90^{\circ}$. The elastic behavior of grains has in fact a negligible influence still at such small strain. Indeed, as shown in Figure 4, creep would be almost in the stationary regime at $\bar{\varepsilon}_{33}=3 . \times 10^{-4}$ if the reference stress of the secondary system would not increase with strain. This result can be also established directly since, in the definition (A3) of the elastoviscoplastic compliance $\mathbf{Q}^{(r)}$, the viscoplastic part $\mathbf{M}^{(r)} t\left(\mathbf{M}^{(r)}\right.$ is of the order of $\left.10^{-4} \mathrm{MPa}^{-1} \mathrm{~s}^{-1}\right)$ quickly dominates over the elastic part $\mathbf{S}^{(r)}\left(\sim 10^{-4} \mathrm{MPa}^{-1}\right)$, say for $t \geq 10 \mathrm{~s}$. The difference between the two distributions at $\bar{\varepsilon}_{33}=3 . \times 10^{-4}$ and $\bar{\varepsilon}_{33}=2 . \times 10^{-2}$ is therefore essentially attributed to the strain hardening of the reference shear stresses of prismatic slip. A similar feature has been observed for the creep of zirconium alloys [Brenner et al., 2002a] which are made, as ice, from hexagonal grains with high viscoplastic anisotropy.

[30] The distribution of deformation in the different grain orientations can be further clarified by the investigation of the intraphase strain heterogeneities. Figure 8 


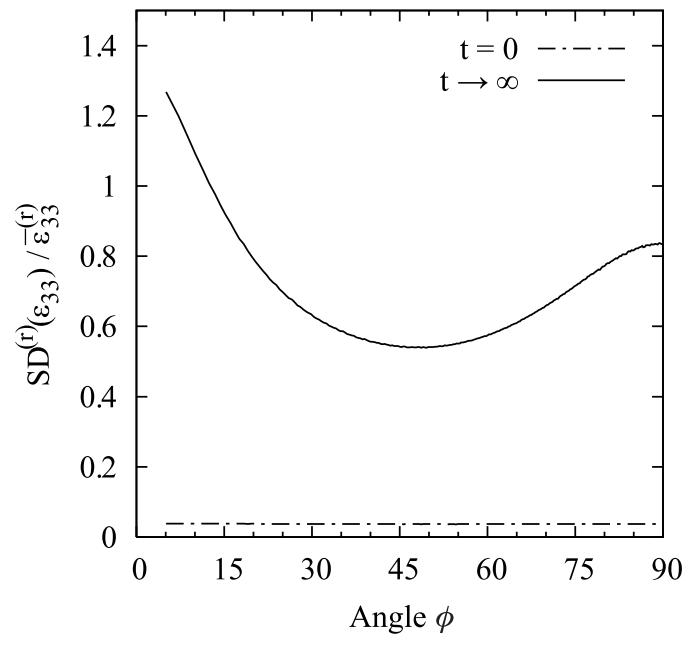

Figure 8. Distribution of the normalized standard deviation $\mathrm{SD}^{(r)}\left(\varepsilon_{33}\right) / \bar{\varepsilon}_{33}{ }^{(r)}$ of the axial strain with respect to the angle $\phi$ between the macroscopic compression direction and the $c$ axis upon loading $(t=0)$ and for the permanent creep regime $(t \rightarrow \infty)$.

shows the distribution of the standard deviation of $\varepsilon_{33}$, defined as

$$
\mathrm{SD}^{(r)}\left(\varepsilon_{33}\right)=\sqrt{\left\langle\varepsilon_{33}^{2}(\mathbf{x})\right\rangle^{(r)}-\left[\left\langle\varepsilon_{33}(\mathbf{x})\right\rangle^{(r)}\right]^{2}}
$$

and normalized by the phase average deformation $\bar{\varepsilon}_{33}^{(r)}=$ $\left\langle\varepsilon_{33}\right\rangle^{(r)}$. This quantity reflects the fluctuations of the strain component $\varepsilon_{33}$ within the orientation $(r)$. Since it is an average over grains with lattice orientation $(r)$, it accounts for the strain fluctuation inside each of those grains, such as the strain localization at grain boundaries often observed in numerical and experimental studies. Recall that within homogenization scheme, an orientation $(r)$ comprises an infinite number of grains with different surrounding and different shape. The standard deviation calculated here thus takes into account the complete intergranular interaction. It must be made clear, however, that long-range interactions associated with spatial correlations between dislocation avalanches are not considered owing to the simplicity of the adopted local constitutive relation (5). For purely elastic and purely viscoplastic regime, the standard deviations (8) can be calculated by an appropriate differentiation of the corresponding effective potential [Brenner et al., 2004].

[31] Results are thus shown at $t=0 \mathrm{~s}$ (instantaneous loading, elastic response) and at $t \rightarrow \infty$ (steady state, viscoplastic response). According to Figure 8 , the normalized strain fluctuation $\mathrm{SD}^{(r)}\left(\varepsilon_{33}\right) / \bar{\varepsilon}_{33}^{(r)}$ in the purely elastic regime is almost independent on $\phi$, and exhibits a low level of $\sim 0.07$. Again, this is attributed to the low elastic anisotropy of grains leading to a weak grain interaction, and an almost uniform strain (and stress) state throughout the material. Results in the viscoplastic regime depart significantly from the elastic one. First of all, the overall level is very high, about 0.8 . This means that at some specific locations, the local strain $\varepsilon_{33}(\mathbf{x})$ can be as high as 2-3 times larger than the phase average one $\bar{\varepsilon}_{33}^{(r)}$, and sometimes, it may even be of opposite sign, in agreement with full-field computations on 2-D ice [Lebensohn et al., 2008]. Moreover, it can be observed that the normalized standard deviation $\mathrm{SD}^{(r)}\left(\varepsilon_{33}\right) / \bar{\varepsilon}_{33}^{(r)}$ presents a minimum at $\phi \simeq 45^{\circ}$, which corresponds to the soft grain orientation. Those grains thus not only deform more rapidly, but also more homogeneously (for a given level of local strain), than the rest of the polycrystal. However, since the average strain $\bar{\varepsilon}_{33}^{(r)}$ is far larger in those lattice orientations, the unnormalized value $\operatorname{SD}^{(r)}\left(\varepsilon_{33}\right)$ is maximal at $\phi \simeq 45^{\circ}$. Strain gradient, and thus probably also intragranular lattice misorientation and strain energy, are thus expected to be larger at $\phi \simeq 45^{\circ}$. Interestingly, these are the only orientations that remain after migration recrystallization has occurred, probably associated to the preferential nucleation and also the preferential growth of grains of that orientation in the presence of long-range internal stress field. Finally, in the transient regime, it is expected that strain distribution obeys a distribution close to the one obtained in the permanent creep regime, even at small overall strain, as discussed above. The large and rapid decrease of the overall strain rate in the transient regime is thus associated with the strong build up of grain interaction and intergranular and intragranular stress and strain heterogeneities.

\subsection{Implications for Slip Systems}

[32] The approach followed in this work relies on the assumption that ice deforms by slip on basal and nonbasal planes. Prismatic slip is in accordance with the presence of short edge segments of dislocations on the prismatic plane, as seen in ice single crystals [Higashi et al., 1985]. Deformation along the $c$ axis requires other slip systems such as the pyramidal slip. Dislocations observed in ice single crystals are not glissile on this slip plane [Hondoh, 2000]. Other deformation modes associated with basal slip can be invoked. As mentioned above, grain boundary migration and grain boundary sliding could accommodate basal slip by making compatible the deformation between grains. But these mechanisms appear to be efficient only at low stress level corresponding to a stress exponent lower than 2 for the polycrystal. Another possibility is the role of deformation inhomogeneities within grains as observed by X-ray diffraction on single crystal [Montagnat et al., 2003a, $2003 \mathrm{~b}$ ]. The interactions between grains at the origin of the large geometric transient creep are obviously dependent on the development of strain heterogeneities within grains. On the other hand, the bending of basal planes associated with basal edge dislocations could contribute to the necessary small deformation along the $c$ axis. Concerning the hardening of prismatic slip systems introduced to reproduce the transient creep, it could be related to the interaction at short distance between nonbasal edge dislocations produced by cross slip or the climb of basal dislocations. Indeed, X-ray diffraction experiments [Montagnat et al., 2006] together with finite element modeling [Taupin et al., 2007] have demonstrated that internal stresses can initiate nonbasal slip in single crystal well oriented for basal slip. In such configurations, the nonbasal slip allows a softening of the basal system, and acts as an efficient mechanism of basal dislocation multiplication, while the nonbasal system is hardened by jog interactions with dislocations. 


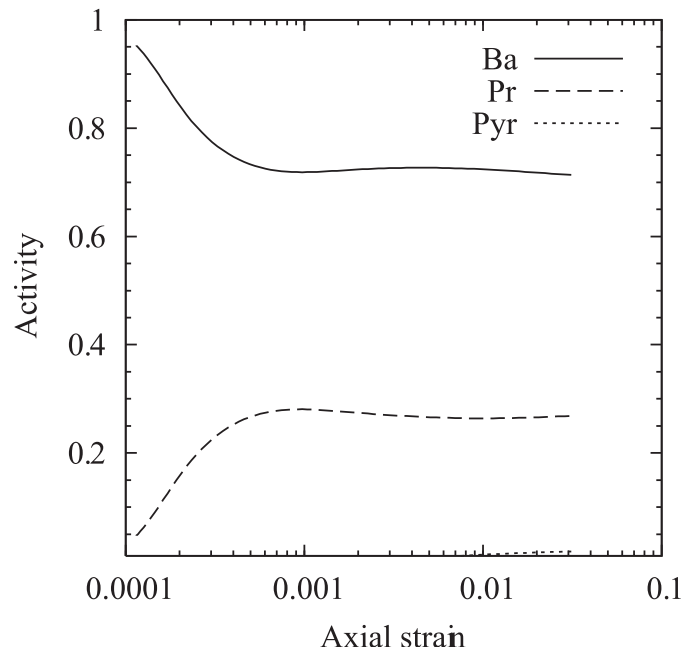

Figure 9. Evolution during transient creep of the relative contribution to the overall plastic deformation of the basal, prismatic, and pyramidal slip systems. Pyramidal activity is always less than $2 \%$.

[33] As discussed above, the physical relevance of pyramidal slip that had to be introduced here to reproduce the correct anisotropy of GRIP specimen may be addressed. We have plotted in Figure 9 the relative contribution to the overall strain of the different slip families, during transient regime. Basal slip is clearly dominant, in agreement with experimental observations. At low strain, only basal slip is activated owing to the almost uniform stress distribution in the different lattice orientations. As deformation, proceeds, the stress is redistributed to the harder grains, leading to an increase activation of nonbasal slip systems. However, it is worth noting that among nonbasal systems, only prismatic slip contributes significantly to the overall deformation. The activity of pyramidal slip is extremely small, less than $\sim 2 \%$. This latter mechanism may thus represent the effect of secondary accommodation processes as described above, but only shows a very minor contribution to the overall deformation. But it is emphasized that its introduction is necessary to obtain the correct anisotropy description for textured specimen, as shown in Figure 3. Note also that pyramidal slip is here the only system with a stress sensitivity $n>3$, and therefore it is of high importance to reproduce the correct overall viscoplastic behavior. Without this system, the overall stress sensitivity would be significantly underestimated. A similar dependence between the overall stress sensitivity and the rheology of the hard slip systems has been reported for zirconium alloys [Brenner et al., 2002a]. Finally, it is worth noting that the activity of basal and prismatic slip does not evolve at strain larger than $\sim 5 \times 10^{-4}$, even if the overall strain rate continues decreasing by more than 1 order of magnitude (see Figure 5). The hardening of the secondary systems thus does not affect significantly their activity after some moderate strain, but only contributes to the overall hardening of the polycrystal.

[34] Finally, to assess the mechanical relevance of the considered slip system, we compare in Figure 10 the viscoplastic response of single crystal used here to the experi- mental data basis on permanent creep compiled by Duval et al. [1983]. Results are shown for crystals compressed at $45^{\circ}$ from the $c$ axis, promoting thus basal slip, but also for compression along or perpendicular to the $c$ axis, promoting respectively pyramidal and prismatic slip. It can be observed that the considered grain rheology is qualitatively correct, with about 4 orders of magnitude difference between basal and nonbasal strain rates. However, slip on nonbasal plane is apparently still not hard enough, since it is about 1-2 orders of magnitude softer than estimated from mechanical tests on single crystals. We cannot completely exclude that at least part of this apparent underestimation of the nonbasal reference stresses is attributed to the affine linearization scheme, which is known to overestimate the effective flow stress [Bornert et al., 2001; Masson et al., 2000; Castelnau et al., 2008a], and in particular at high local anisotropy as considered here. But, in any case, the cross slip of basal dislocations on prismatic planes is associated with the long-range internal stress field produced by basal dislocations [Montagnat et al., 2006]. Therefore, this deformation process is made very difficult when single crystals are deformed in compression along or perpendicular to the $c$ axis since basal slip is avoided. As a consequence, it is expected that the resistance of prismatic and pyramidal slip deduced from the deformation of single crystals must be greater than those deduced from the response of polycrystals as given in Table 1, in good agreement with our results.

[35] It is also worth noting that the single-crystal rheology identified here is believed to be more reliable than the earlier estimation used by Castelnau et al. [1996, 1997, 1998], due to some inconsistencies of the earlier "tangent" SC model used in those papers, already pointed out by Masson et al. [2000], and partially corrected in the present affine SC model. In particular, the "tangent" method was limited to a unique stress sensitivity for all slip systems. With the present affine model, we choose $n_{(\mathrm{Ba})}=2$, and we

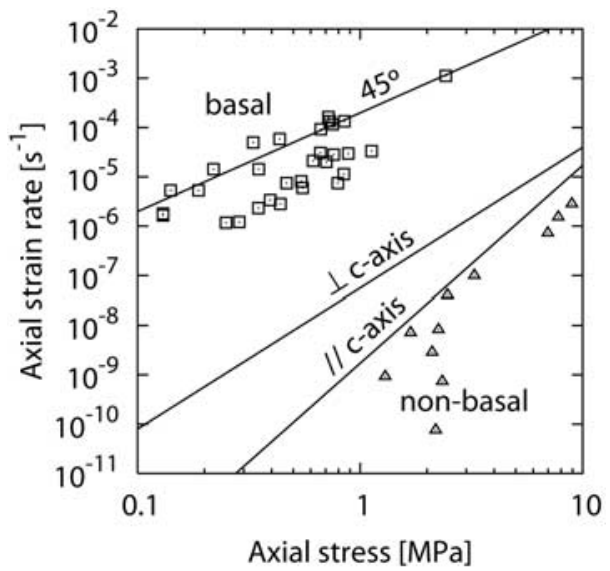

Figure 10. Stationary creep behavior of single crystals at $-10^{\circ} \mathrm{C}$, given by the present model (lines) and compared to the data set compiled by Duval et al. [1983] (points). Results are indicated for compression at $45^{\circ}$ from the $c$ axis (activation of basal slip), as well as for compression perpendicular (activation of prismatic slip) and parallel (activation of pyramidal systems) to the $c$ axis. 
identified larger values for $n_{(\mathrm{Pr})}$ and $n_{(\mathrm{Pyr})}$, in good agreement with experiment data on single crystals and polycrystals.

\section{Concluding Remarks}

[36] We have presented the first micromechanical modeling of the elastoviscoplastic behavior of polycrystalline ice. The model used here is based on the mean-field selfconsistent theory, which aims at bridging scales between the behavior of the ice crystal and the behavior of the polycrystal. The procedure accounts for the long-term memory effect, characterized by the fact that the actual stress and strain (rate) fields in the material depend on the whole loading history of the specimen. This feature is a consequence of the dependence of the strain rate on both stress and stress rate. The treatment of the nonlinear character of the viscoplastic behavior was handled with the "affine" procedure, with provides a good compromise between accuracy and simplicity. Information on intragranular strain heterogeneities could be also deduced from this model. A simple phenomenological rheological law was adopted for the viscoplastic behavior at the local (grain) scale, with easy (dislocation) glide on the basal planes, and higher resistance on secondary systems, whereas strain hardening/softening was introduced at the slip system level depending on the local strain rate configuration. This local constitutive relation was identified on experimental data on single and poly crystal data (transient and permanent creep regimes).

[37] One salient conclusion coming out from the presented results is the ability of the model, in spite of its relative simplicity, to reproduce the essential features of ice rheology. In the steady state regime, when using singlecrystal rheology that lies close to the experimental one, the model well reproduces the overall stress sensitivity, the overall flow stress of isotropic specimens, and the anisotropy of polar ices exhibiting a large variety of Lattice Preferred Orientations. This shows that the mechanical effects of the intergranular interactions are correctly treated, and that the model lies on a robust physical basis. The introduction of prismatic and pyramidal slip systems, which relevance has been discussed, is required to obtain a good agreement with experiments. It is remarkable that the overall stress sensitivity is highly influenced by stress sensitivity of the pyramidal system, even if this system does not contribute by more than $2 \%$ to the total strain rate. Concerning the transient creep of isotropic ice polycrystals, the model accurately predicts the strong overall hardening with a strain rate decreasing by more than 2 orders of magnitude. This effect is attributed to the stress redistribution in the material (kinematic hardening), from an almost uniform stress state upon loading, due to the small elastic anisotropy of grains, to a largely heterogeneous distribution as soon as some plastic deformation has taken place. The importance of the transient creep in the deformation of the polycrystal is the consequence of the very strong viscoplastic anisotropy of the ice crystal. We have shown that grains with $c$ axis oriented at $45^{\circ}$ from the macroscopic compression axis deform more rapidly, under a lower stress level, and exhibit a larger strain heterogeneity than other grain orientations. The hardening rate of prismatic slip in the transient creep, which may represent the nonbasal dislocation glide and the interaction of the nonbasal jogs with basal dislocations [Taupin et al., 2007], is essential to explain that the minimum creep rate occurs at a strain of $1 \%$.

[38] Evidently, there is room to improve this first application. Concerning the scale transition scheme, the used affine method is known to provide a too stiff estimation of the overall (nonlinear) viscoplastic behavior. More recently, the so-called second-order procedure has been proposed for purely viscoplastic behavior [Ponte Castañeda, 2002]. It has been applied to several crystalline structure including strongly anisotropic materials [Lebensohn et al., 2007] and olivine for thermomechanical conditions relevant for the Earth upper mantle [Castelnau et al., 2008a]. The superiority of this procedure has been attested by comparison with reference results obtained by full-field methods. A first attempt to extend this method to elastoviscoplasticity has been provided by Brenner and Masson [2005], whereas promising and theoretically more rigorous developments have been proposed very recently [Lahellec and Suquet, 2007b]. One may expect from an application to polar ices that the resistance of prismatic and pyramidal slip systems can be taken even larger than the one considered in the present study, and therefore one may expect an even better agreement with the experimental data. In this context and considering the large viscoplastic anisotropy of the crystal, ice is taken as a model material to validate micro-macro approaches used to estimate the behavior of viscoplastic materials [Gilormini et al., 2001; Lebensohn et al., 2004].

[39] Next, it would be of interest to test the model on more complex loading conditions, e.g., on samples submitted to successive loading and (partial) unloading sequences, for various stress levels. Particularly interesting would be to investigate the recoverable part of the transient creep due to the long-range internal stress field that develops during deformation and which is at the origin of the significant kinematic hardening. Such a procedure would of course provide an even stronger validation of the model, but also a deeper understanding of ice rheology. This requires further numerical developments of the model that will be addressed in future work.

\section{Appendix A: Elastoviscoplastic Micromechanical Modeling}

[40] Compared to the simpler cases of purely elastic or purely viscoplastic polycrystals, the main difficulty for estimating the effective behavior of elastoviscoplastic polycrystals comes from the fact that both stress and stress rate enter in the constitutive relation at the grain scale. Consequently, the local mechanical response at time $t$ depends on the entire loading history of the specimen. A specific treatment is thus required. In the following, the main assumptions of mean-field homogenization procedures, and of the affine SC model from Masson and Zaoui [1999] with some simplifications for the calculations of the inverse Laplace transforms, are briefly recalled. For reasons that will become evident in the sequel, we start with the homogenization of linear elastoviscoplastic polycrystals, i.e., for which $n_{(k)}=1 \forall k$.

\section{A1. Exact Solution for Linear Elastoviscoplasticity}

[41] Exact solutions can be theoretically derived in the case of polycrystals exhibiting a linear viscosity. The 
constitutive relation (2) can be expressed in the general form

$$
\dot{\varepsilon}(\mathbf{x}, t)=\mathbf{S}^{(r)}: \dot{\boldsymbol{\sigma}}(\mathbf{x}, t)+\mathbf{M}^{(r)}: \boldsymbol{\sigma}^{\prime}(\mathbf{x}, t)+\dot{\varepsilon}_{0}^{(r)}(t)
$$

with $\mathbf{M}^{(r)}$ and $\dot{\varepsilon}_{0}^{(r)}$ denoting the viscous compliance and a stress free strain rate respectively. We emphasize that in equation (A1), $\mathbf{M}^{(r)}$ and $\dot{\varepsilon}_{0}^{(r)}$ are both assumed to be uniform in grains with lattice orientation $(r)$. Without loss of generality, the integration of the above equation leads to the following expression for the local total strain

$$
\varepsilon(\mathbf{x}, t)=\mathbf{Q}^{(r)}(t) * \boldsymbol{\sigma}(\mathbf{x}, t)+\varepsilon_{0}^{(r)}(t),
$$

where the asterisk denotes the Stieljes convolution product. The time-dependent elastoviscoplastic compliance $\mathbf{Q}^{(r)}$ depends on both the elastic and viscoplastic compliances at the grain scale

$$
\mathbf{Q}^{(r)}(t)=\mathbf{S}^{(r)}+\mathbf{M}^{(r)} t .
$$

Note that $\mathbf{Q}^{(r)}$ is also uniform in orientation $(r)$. Now, the homogenization problem can be solved by means of the correspondence principle [Mandel, 1966; Laws and McLaughlin, 1978]. Since the Laplace-Carson transform acts on time variables $t$ and not on space variables $\mathbf{x}$, the mechanical problem can be Laplace transformed without loss of generality. Equation (A2) gives

$$
\hat{\boldsymbol{\varepsilon}}(\mathbf{x}, p)=\hat{\mathbf{Q}}^{(r)}(p): \hat{\boldsymbol{\sigma}}(\mathbf{x}, p)+\hat{\varepsilon}_{0}^{(r)}(p)
$$

with hat denoting Laplace-Carson transformed quantities and $p$ denoting the (complex) Laplace variable. The convolution product in (A2) has become a direct product in (A4). The local constitutive relation (A4) in the LaplaceCarson space has thus a form similar as a standard linear thermoelastic behavior, for which homogenization results are well established. The effective behavior of the polycrystal reads

$$
\hat{\bar{\varepsilon}}(p)=\hat{\tilde{\mathbf{Q}}}(p): \hat{\overline{\boldsymbol{\sigma}}}(p)+\hat{\tilde{\boldsymbol{\varepsilon}}}_{0}(p)
$$

with $\bar{\sigma}$ and $\bar{\varepsilon}$ the effective stress and strain respectively, given by the volume average (denoted by angle brackets) of the corresponding local quantities

$$
\overline{\boldsymbol{\sigma}}(t)=\langle\boldsymbol{\sigma}(\mathbf{x}, t)\rangle, \quad \overline{\boldsymbol{\varepsilon}}(t)=\langle\varepsilon(\mathbf{x}, t)\rangle .
$$

The effective elastoviscoplastic modulus $\tilde{\mathbf{Q}}$ and effective stress-free strain $\tilde{\varepsilon}_{0}$ are given in the Laplace-Carson space by the following exact relations

$$
\hat{\tilde{\mathbf{Q}}}=\left\langle\hat{\mathbf{Q}}^{(r)}: \hat{\mathbf{B}}\right\rangle, \quad \hat{\tilde{\varepsilon}}_{0}=\left\langle\hat{\varepsilon}_{0}^{(r)}: \hat{\mathbf{B}}\right\rangle,
$$

where $\mathbf{B}$ is the stress localization tensor relating the local stress tensor to the macroscopic one. In this context, the SC scheme provides an exact estimate for the phase average value of $\mathbf{B}$ for materials exhibiting a "perfectly disordered" microstructure [Kröner, 1978] (exact solution means that stress equilibrium, strain compatibility, and boundary conditions are fulfilled in a statistical way, together with the local constitutive relation). The SC scheme is therefore especially appropriate for polycrystals. The expression of the stress localization tensor in a general context of anisotropy is given, e.g., by Brenner et al. [2004].

[42] Once the homogenization has been carried out in the Laplace-Carson space, results have to be back-transformed in the physical space for an easier mechanical interpretation. This last difficulty can be quite cumbersome for the numerical implementation of the method although some solutions have been proposed and applied [Schapery, 1962; Turner and Tomé, 1993]. Here, we use an approximate inversion adapted by Brenner et al. [2002b] which is particularly well suited for the investigation of the (monotone) creep behavior of polycrystals. However, this approximation prevents the model to be applied to more complex loading paths, e.g., for the study of recoverable strain shown in Figure 1 as also discussed by Turner et al. [1994].

[43] It can be easily seen that upon creep test with an instantaneous loading at $t=0$, two limit regimes can be identified. At $t=0$, since $\mathbf{Q}^{(r)}=\mathbf{S}^{(r)}$, the instantaneous response of the material is purely elastic, and the stress distribution in the different grain orientations only depends on the local elastic anisotropy. On the other hand, for $t \rightarrow$ $\infty$, the compliance $\mathbf{Q}^{(r)} \rightarrow \mathbf{M}^{(r)} t$. The permanent creep regime is therefore insensitive to the elastic response of grains. In that latter case, the stress and strain rate distribution only depends on the local viscoplastic anisotropy.

[44] Finally, it is worth emphasizing that in the above treatment, the local stress and strain (rate) have not been assumed to follow any specific spatial distribution. Recall that an orientation $(r)$ (or mechanical phase) comprises many grains with the same crystallographic orientation but exhibiting different morphologies and surroundings. In those grains, the stress and strain are naturally heterogeneous. The mean-field SC approach does not allow to access the value of stress and strain at a specific spatial position $\mathbf{x}$, but only to characterize the stress and strain distribution within a phase $(r)$. The use of the Eshelby [1957] formalism in the SC formulation allows to solve for the phase average stress and strain when the average grain shape is ellipsoidal

$$
\overline{\boldsymbol{\sigma}}^{(r)}=\langle\boldsymbol{\sigma}(\mathbf{x})\rangle^{(r)}, \quad \overline{\boldsymbol{\varepsilon}}^{(r)}=\langle\varepsilon(\mathbf{x})\rangle^{(r)} .
$$

Note again that this does not mean that those quantities are assumed to be uniform in the orientation $(r)$. Indeed, the heterogeneity of stress and strain rate within a given lattice orientation (so-called "intraphase" heterogeneity) can be partially characterized by an appropriate derivation of the effective potentials [Bobeth and Diener, 1987; Kreher, 1990; Brenner and Masson, 2005].

\section{A2. Extension to Nonlinear Elastoviscoplasticity}

[45] The additional difficulty when dealing with material exhibiting a nonlinear viscoplastic behavior is that the local viscosity is stress-dependent. Consequently, since the stress is generally not uniformly distributed in a given lattice orientation, the local constitutive relation cannot be written in a form analog to (A1) with phase uniform $\mathbf{M}^{(r)}$ and $\dot{\varepsilon}_{0}^{(r)}$. The classical treatment to bypass this difficulty is to introduce a multiphase "Linear Comparison Polycrystal" (LCP) that has the same microstructure as the real nonlinear 
polycrystal, and for which well-known homogenization results apply. The "affine" method proposed by Masson and Zaoui [1999] consists in a tangent linearization of relation (5) with respect to the phase average shear stress on system $(k)$. The local behavior of system $(k)$ for a linearization time $\xi$ reads [Rougier et al., 1994]

$$
\dot{\gamma}_{(k)}(\mathbf{x}, t)=\alpha_{(k)}^{(r)}(\xi) \tau_{(k)}(\mathbf{x}, t)+\dot{e}_{(k)}^{(r)}(t, \xi)
$$

with

$$
\alpha_{(k)}^{(r)}(\xi)=\left.\frac{\partial \dot{\gamma}_{(k)}}{\partial \tau}\right|_{\tau=\left\langle\tau_{(k)}(\xi)\right\rangle^{(r)}},
$$

where $\dot{\gamma}_{(k)}(\tau)$ denotes the shear rate on system $(k)$ given by the nonlinear relation (5) for the shear stress $\tau_{(k)}$, and

$$
\begin{array}{cc}
\dot{e}_{(k)}^{(r)}(t, \xi)=\dot{\gamma}_{(k)}(\tau(t))-\alpha_{(k)}^{(r)}(\xi) \tau_{(k)}(t) & \forall t \leq \xi \\
\dot{e}_{(k)}^{(r)}(t, \xi)=\dot{\gamma}_{(k)}(\tau(t))-\alpha_{(k)}^{(r)}(\xi) \tau_{(k)}(\xi) & \forall t \geq \xi .
\end{array}
$$

The (linearized) viscoplastic compliance and stress free strain rate finally read

$$
\begin{gathered}
\mathbf{M}^{(r)}(\xi)=\sum_{k} \alpha_{(k)}^{(r)}(\xi) \boldsymbol{\mu}_{(k)}^{(r)} \otimes \boldsymbol{\mu}_{(k)}^{(r)} \\
\dot{\varepsilon}_{0}^{(r)}(t, \xi)=\sum_{k} \dot{e}_{(k)}^{(r)}(t, \xi) \boldsymbol{\mu}_{(k)}^{(r)}
\end{gathered}
$$

with $\otimes$ denoting the dyadic product. This allows the homogenization treatment introduced in section A2 to be applied at any time $t=\xi$. Eventually, it is emphasized that the obtained effective behavior remains nonlinear because of the dependence of the linearized compliances on the actual stress, as shown by equations (A10) and (A11).

[46] Acknowledgments. The sabbatical leave of O. Castelnau at IGPP was funded by CNRS and the Green Foundation at IGPP/SIO. This work was also supported by CNRS (Department ST2I).

\section{References}

Ahmad, S., and R. W. Whitworth (1988), Dislocation motion in ice: a study by synchroton X-ray topography, Philos. Mag. A, 57(5), 749-766.

Alley, R. B. (1992), Flow-law hypotheses for ice sheet modeling, J. Glaciol., 38, 245-256.

Andrade, E. (1910), On the viscous flow in metals and allied phenomena, Proc. R. Soc. London, Ser. A, 84, 1-12.

Ashby, M. F., and P. Duval (1985), The creep of polycrystalline ice, Cold Reg. Sci. Technol., 11, 285-300.

Barbot, S., Y. Hamiel, and Y. Fialko (2008), Space geodetic investigation of the coseismic and postseismic deformation due to the $2003 M_{w} 7.2$ Altai earthquake: Implications for the local lithospheric rheology, J. Geophys. Res., 113, B03403, doi:10.1029/2007JB005063.

Blackman, D. K. (2007), Use of mineral physics, with geodynamic modeling and seismology, to investigate flow in the Earth's mantle, Rep. Prog. Phys., 70, 659-689.

Bobeth, M., and G. Diener (1987), Static and thermoelastic field fluctuations in multiphase composites, J. Mech. Phys. Solids, 35, 137-149.

Bornert, M., R. Masson, P. Ponte Castañeda, and A. Zaoui (2001), Secondorder estimates for the effective behaviour of viscoplastic polycrystalline materials, J. Mech. Phys. Solids, 49, 2737-2764.

Brenner, R., and R. Masson (2005), Improved affine estimates for nonlinear viscoelastic composites, Eur. J. Mech. A Solids, 24, 1002-1015.

Brenner, R., J.-L. Béchade, O. Castelnau, and B. Bacroix (2002a), Thermal creep of Zr-Nb1\%-O alloys: experimental analysis and micromechanical modelling, J. Nucl. Mater., 305, 175-186.
Brenner, R., R. Masson, O. Castelnau, and A. Zaoui (2002b), A “quasielastic" affine formulation for the homogenized behaviour of nonlinear viscoelastic polycrystals and composites, Eur. J. Mech. A Solids, 21, 943-960.

Brenner, R., O. Castelnau, and L. Badea (2004), Mechanical field fluctuations in polycrystals estimated by homogenization techniques, Proc. $R$. Soc. London, Ser A, 460, 3589-3612.

Bronkhorst, C. A., S. R. Kalidindi, and L. Anand (1992), Polycrystalline plasticity and the evolution of crystallographic texture in fcc metals, Proc. $R$ Soc. London, Ser A, 341, 443-477.

Castelnau, O., P. Duval, R. A. Lebensohn, and G. R. Canova (1996), Viscoplastic modelling of texture development in polycrystalline ice with a self- consistent approach; comparison with bound estimates, J. Geophys. Res., 101, 13,851-13,868.

Castelnau, O., G. Canova, R. Lebensohn, and P. Duval (1997), Modelling viscoplastic behavior of anisotropic polycrystalline ice with a selfconsistent approach, Acta Mater., 45, 4823-4834.

Castelnau, O., H. Shoji, A. Mangeney, H. Milsch, P. Duval, A. Miyamoto, K. Kuwabara, and O. Watanabe (1998), Anisotropic behavior of GRIP ices and flow in central Greenland, Earth Planet. Sci. Lett., 160, 1-13.

Castelnau, O., H. Francillette, B. Bacroix, and R. A. Lebensohn (2001), Texture dependent plastic behavior of zr 702 at large strain, J. Nucl. Mater., 297, 14-26.

Castelnau, O., R. Brenner, and R. Lebensohn (2006), The effect of strain heterogeneity on the work-hardening of polycrystals predicted by meanfield approaches, Acta Mater, 54, 2745-2756.

Castelnau, O., D. K. Blackman, R. A. Lebensohn, and P. Ponte Castañeda (2008a), Micromechanical modeling of the viscoplastic behavior of olivine, J. Geophys. Res., 113, B09202, doi:10.1029/2007JB005444.

Castelnau, O., R. A. Lebensohn, Ponte CastañP. eda, and D. K. Blackman (2008b), Earth mantle rheology inferred from homogenization theories, in Multiscale Modeling of Heterogenous Materials: From Microstructure to Macro-scale Properties, edited by O. Cazacu, pp. 55-70, ISTE, London.

Cottrell, A. (1996), Andrade creep, Philos. Mag. Lett., 75, 35-37.

Cottrell, A. (1997), Logarithmic and Andrade creep, Philos. Mag. Lett., 75 $301-307$.

Dahl-Jensen, D., and N. S. Gundestrup (1987), Constitutive properties of ice at Dye 3, Greenland, in Physical Basis of Ice Sheet Modeling, IAHS Publ. 170, 31-43.

Dawson, P. R., and H. R. Wenk (2000), Texturing of the upper mantle convection, Philos. Mag. A., 80, 573-598.

Duval, P. (1976), Lois du fluage transitoire ou permanent de la glace polycristalline pour divers états de contrainte, Ann. Geophys., 32(4), 335350

Duval, P. (1978), Anelastic behavior of polycrystalline ice, J. Glaciol., 21, $621-628$.

Duval, P., and O. Castelnau (1995), Dynamic recrystallization of ice in polar ice sheets, J. Physique IV, 3(5), 197-205.

Duval, P., M. F. Ashby, and I. Anderman (1983), Rate-controlling processes in the creep of polycrystalline ice, J. Phys. Chem., 87(21), 4066-4074.

Dysthe, D., Y. Podladchikov, F. Renard, J. Feder, and B. Jamtveit (2002), Universal scaling in transient creep, Phys. Rev. Lett., 89, 246102, doi:10.1103/PhysRevLett.89.246102.

Eshelby, J. D. (1957), The determination of the elastic field of an ellipsoidal inclusion, and related problems, Proc. R. Soc. London, Ser. A, 241, 376396.

Friedel, J. (1964), Dislocations, Pergamon, New York.

Gammon, P. H., H. Kiefte, M. J. Clouter, and W. W. Denner (1983), Elastic constants of artificial and natural ice samples by brilloin spectroscopy, J. Glaciol., 29(103), 433-460.

Gilormini, P., M. V. Nebozhyn, and P. Ponte Castañeda (2001), Accurate estimates for the creep behavior of hexagonal polycrystals, Acta Mater. $49,329-337$.

Goldsby, D., and D. Kohlstedt (2001), Superplastic deformation of ice: experimental observations, J. Geophys. Res., 106, 11,017-11,030.

Gow, A. J., and T. Williamson (1976), Rheological implications of the internal structure and crystal fabrics of the West Antarctic ice sheet as revealed by deep core drilling at byrd station, Tech. Rep. 76-35, Cold Reg. Res. and Eng. Lab., Hanover, N. H.

Griggs, D. T., and N. E. Coles (1954), Creep of single crystals of ice, Rep. 11, Cold Reg. Res. and Eng. Lab., Hanover, N. H.

Hershey, A. V. (1954), The elasticity of an isotropic aggregate of anisotropic cubic crystals, J. Appl. Mech., 21, 236-240.

Higashi, A., S. Koinuma, and S. Mae (1964), Plastic yielding in ice crystals, Z. Naturforsch., 19, 459-470.

Higashi, A., A. Fukuda, T. Hondoh, K. Goto, and S. Amakai (1985), Dynamical dislocation processes in ice crystal, in Proceeding of the Yamada Conference IX on Dislocations in Solids, edited by T. Suzuki et al., pp. 511-515, Univ. of Tokyo Press, Tokyo. 
Hondoh, T. (2000), Nature and behavior of dislocations in ice, in Physics of Ice Core Records, edited by T. Hondoh, pp. 3-24, Hokkaido Univ. Press, Sapporo, Japan

Hondoh, T., H. Iwamatsu, and S. Mae (1990), Dislocation mobility for nonbasal glide in ice measured by in situ X-ray topography, Philos. Mag. A, 62(1), 89-102.

Hutchinson, J. W. (1977), Creep and plasticity of hexagonal polycrystals as related to single crystal slip, Metal. Trans. A, 8(9), 1465-1469.

Jacka, T., and L. Jun (2000), Flow rates and crystal orientation fabrics in compression of polycrystalline ice at low temperatures and stresses, in Physics of Ice Core Records, edited by T. Hondoh, pp. 83-102, Hokkaido Univ. Press, Sapporo, Japan.

Jacka, T. H. (1984), The time and strain required for development of minimum strain rates in ice, Cold Reg. Sci. Technol., 8, 261-268.

Karato, S., and H. A. Spetzler (1990), Defect microdynamics in minerals and solid-state mechanisms of seismic wave attenuation and velocity dispersion in the mantle, Rev. Geophys., 28, 399-421.

Kenner, S., and P. Segall (2000), Postseismic deformation following the 1906 San Francisco earthquake, J. Geophys. Res., 109, 13,195-13,209.

Kirby, S. H., W. B. Durham, M. L. Beeman, H. C. Heard, and M. A. Daley (1987), Inelastic properties of ice ih at low temperatures and high pressures, J. Phys. C Solid State Phys., 48(C1), 227-232.

Kocks, U. F. (1976), Laws for work-hardening and low-temperature creep, J. Eng. Mater. Technol., 10, 76-85.

Kreher, W. (1990), Residual stresses and stored elastic energy of composites and polycrystals, J. Mech. Phys. Solids, 38, 115-128.

Kröner, E. (1958), Berechnung der elastischen konstanten des vielkristalls aus den konstanten des einkristalls, Z. Phys., 151, 504-518.

Kröner, E. (1978), Self-consistent scheme and graded disorder in polycrystal elasticity, J. Phys. F Metal Phys., 8, 2261-2267.

Lahellec, N., and P. Suquet (2006), Effective behavior of linear viscoelastic composites: A time-integration approach, Int. J. Solids Struct., 44, 507 529

Lahellec, N., and P. Suquet (2007a), On the effective behavior of nonlinear inelastic composites: I. Incremental variational principles, J. Mech. Phys. Solids, 55, 1932-1963.

Lahellec, N., and P. Suquet (2007b), On the effective behavior of nonlinear inelastic composites: II. A second-order procedure, J. Mech. Phys. Solids, $55,1964-1992$

Laws, N., and R. McLaughlin (1978), Self-consistent estimates for the viscoelastic creep compliances of composite materials, Proc. R. Soc London, Ser. A, 353, 251-273.

Lebensohn, R. A., and C. N. Tomé (1993), A self-consistent anisotropic approach for the simulation of plastic deformation and texture development of polycrystals: Application to zirconium alloys, Acta Metall. Mater. 41(9), 2611-2624.

Lebensohn, R. A., Y. Liu, and P. Ponte Castañeda (2004), On the accuracy of the self-consistent approximation for polycrystals: Comparison with full-field numerical simulations, Acta Mater., 52, 5347-5361.

Lebensohn, R. A., C. N. Tomé, and P. Ponte Castañeda (2007), Selfconsistent modeling of the mechanical behavior of viscoplastic polycrystals incorporating field fluctuations, Philos. Mag., 87(28), 4287-4322.

Lebensohn, R. A., M. Montagnat, P. Mansuy, P. Duval, J. Meysonnier, and A. Philip (2008), Modelling viscoplastic behavior and heterogenous intracrystalline deformation of columnar ice polycrystals, Acta Mater., in press.

Letouzé, N., R. Brenner, O. Castelnau, J-.L. Béchade, and M-.H. Mathon (2002), Residual strain distribution in Zircaloy-4 measured by neutron diffraction and estimated by homogenization techniques, Scr. Mater., 47, $595-599$.

Lipenkov, V., A. Salamatin, and P. Duval (1997), Bubbly-ice densification in ice sheets: Applications, J. Glaciol., 43, 397-407.

Mainprice, D. (1997), Modelling the anisotropic seismic properties of partially molten rocks found at mid-ocean ridges, Tectonophysics, 279, $161-$ 179.

Mandel, J. (1966), Mécanique des Milieux Continus, Gauthier-Villars, Paris, France.

Mangeney, A., and F. Califano (1998), The shallow-ice approximation for anisotropic ice: Formulation and limits, J. Geophys. Res., 103, 691-705.

Mangeney, A., F. Califano, and O. Castelnau (1996), Isothermal flow of an anisotropic ice sheet in the vicinity of an ice divide, J. Geophys. Res., $101,28,189-28,204$

Masson, R., and A. Zaoui (1999), Self-consistent estimates for the ratedependent elastoplastic behaviour of polycrystalline materials, J. Mech. Phys. Solids, 47, 1543-1568.
Masson, R., M. Bornert, P. Suquet, and A. Zaoui (2000), An affine formulation for the prediction of the effective properties of nonlinear composites and polycrystals, J. Mech. Phys. Solids, 48, 1203-1226.

Mellor, M., and R. Testa (1969), Creep of ice under low stress, J. Glaciol., $8,147-152$

Mohit, P. S. (2008), Viscous relaxation and early planetary evolution, Eos Trans., $A G U, 89(20), 185-186$.

Molinari, A., G. R. Canova, and S. Ahzi (1987), A self-consistent approach of the large deformation polycrystal viscoplasticity, Acta Metall., 35(12), 2983-2994.

Montagnat, M., and P. Duval (2000), Rate controlling processes in the creep of polar ice, influence of grain boundary migration associated with recrystallization, Earth Planet. Sci. Lett., 183, 179-186.

Montagnat, M., P. Duval, P. Bastie, and B. Hamelin (2003a), Strain gradients and geometrically necessary dislocations in deformed ice single crystals, Scr Mater. 49, 411-415.

Montagnat, M., P. Duval, P. Bastie, B. Hamelin, and V. Y. Lipenkov (2003b), Lattice distortion in ice crystals from the Vostok core (Antarctica) revealed by hard X-ray diffraction: Implication in the deformation of ice at low stresses, Earth Planet. Sci. Lett., 214, 369-378.

Montagnat, M., J. Weiss, P. Duval, H. Brunjail, P. Bastie, and J. G. Sevillano (2006), The heterogeneous nature of slip in ice single crystals deformed under torsion, Philos. Mag., 86, 4259-4270.

Nebozhyn, M. V., P. Gilormini, and P. Ponte Castañeda (2000), Variational self-consistent estimates for viscoplastic polycrystals with highly anisotropic grains, C. R. Acad. Sci., Ser. IIb, 328, 11-17.

Ponte Castañeda, P. (2002), Second-order homogenization estimates for nonlinear composites incorporating field fluctuations. I-Theory, J. Mech. Phys. Solids, 50, 737-757.

Rougier, Y., C. Stolz, and A. Zaoui (1994), Self-consistent modelling of elastic-viscoplastic polycrystals, C. R. Acad. Sci., 318, 145-151.

Sanchez-Hubert, J., and E. Sanchez-Palencia (1978), Sur certains problèmes physiques d'homogénéisation donnant lieu à des phénomènes de relaxation, C. R. Acad. Sci., Ser. A, 286, 903-906.

Schapery, R. A. (1962), Approximate methods of transform inversion for viscoelastic stress analysis, paper presented at the 14th U.S. National Congress of Theoretical and Applied Mechanics ASME 4th, U.S. Natl. Comm. on Theoret. and Appl. Mech., Blacksburg, Va.

Steinemann, S. (1958), Experimentelle untersuchungen zur plastizität von eis, Beitr. Geol. Schweiz, 10, 1-72.

Suquet, P. (1987), Elements of homogenization for inelastic solid mechanics, in Homogenization Techniques for Composite Media, edited by E. SanchezPalencia and A. Zaoui, pp. 193-278, Springer, Berlin.

Taupin, V., S. Varadhan, J. Chevy, C. Fressengeas, A. Beaudoin, M. Montagnat, and P. Duval (2007), Effects of size on the dynamics of dislocations in ice single crystals, Phys. Rev. Lett., 99, 155,507.

Thorsteinsson, T., J. Kipfstuhl, and H. Miller (1997), Textures and fabrics in the GRIP ice core, J. Geophys. Res., 102, 26,583-26,599.

Turner, P. A., and C. N. Tomé (1993), Self-consistent modeling of viscoelastic polycrystals: application to irradiation creep and growth, J. Mech. Phys. Solids, 41(7), 1191-1211.

Turner, P. A., C. N. Tomé, and C. Woo (1994), Self-consistent modelling of nonlinear visco-elastic polycrystals: An approximate scheme, Philos. Mag. A., 70(4), 689-711.

Weertman, J. (1973), Creep of ice, in Physics and Chemistry of Ice, edited by E. Whalley, S. J. Jones, and L. W. Gold, pp. 320-337, R. Soc. of Can., Ottawa.

Willis, J. R. (1981), Variational and related methods for the overall properties of composites, Adv. Appl. Mech., 21, 2-78.

Yuen, D. A., R. C. A. Sabadini, P. Gasperini, and E. Boschi (1986), On transient rheology and glacial isostasy, J. Geophys. Res., 91, 11,42011,438

R. Brenner and O. Castelnau, Laboratoire des Propriétés Mécaniques et Thermodynamiques des Matériaux, CNRS, Université Paris Nord, avenue J. B. Clément, F-93430 Villetaneuse, France. (rb@1pmtm.univ-paris13.fr; oc@1pmtm.univ-paris13.fr)

P. Duval and M. Montagnat, LGGE-CNRS, Université Joseph Fourier, rue Molière, B.P. 96, F-38402 Saint-Martin d'Hères, France. (duval@ lgge.obs.ujf-grenoble.fr; maurine@lgge.obs.ujf-grenoble.fr) 